\title{
Equivalencia Factorial de la Escala de Actitudes hacia la Autoridad Institucional en Adolescentes (AAI-A) Chilenos y Colombianos
}

\author{
Factorial Equivalence of the Attitudes to Institutional Authority in Adolescence Scale \\ (AAI-A) among Chileans and Colombian Teenagers
}

\author{
José Luis Gálvez-Nieto ${ }^{1}$, Sonia Salvo ${ }^{2}$, Ítalo Trizano-Hermosila ${ }^{3}$, Christian Hederich ${ }^{4}$ y Karina \\ Polanco 5
}

\section{Resumen}

El propósito de esta investigación fue estudiar la equivalencia y estabilidad psicométrica de la Escala de Actitudes hacia la Autoridad Institucional $(A A I-A)$ en adolescentes chilenos y colombianos. Se realizó un estudio de corte transversal en el que participaron dos muestras independientes de adolescentes de ambos sexos, de edades comprendidas entre 12 y 20 años. La primera muestra estuvo compuesta por 692 adolescentes chilenos, y la segunda por 541 adolescentes colombianos. Los resultados obtenidos mediante los procedimientos de validez cruzada, análisis factorial exploratorio y confirmatorio, análisis de invarianza factorial, los análisis correlacionales para evaluar la validez de criterio externo y los análisis de consistencia interna y homogeneidad, permiten concluir que la $A A I-A$ es un instrumento válido y fiable en ambos contextos.

Palabras clave: actitud hacia la autoridad, adolescencia, invarianza factorial

\begin{abstract}
The objective of this research is to study the equivalence and psychometric stability of the scale of attitudes towards authority in Chilean and Colombian teenagers. We carried on a transversal study with two independent samples of teenagers from both gender, aged 12 to 20 years old. The first sample was composed of 692 Chilean teenagers and the second one was composed of 541 Colombian teenagers. The results obtained using cross-validation procedures, exploratory and confirmatory factor analysis, factorial invariance analysis, correlational analyzes to evaluate the validity of external criteria and analyzes of internal consistency and homogeneity allowed us to conclude that $A A I-A$ is an instrument valid and reliable in both contexts.
\end{abstract}

Keywords: attitude toward authority, adolescence, factorial invariance

Agradecimientos: Este artículo reporta resultados parciales del proyecto FONDECYT N 11150157 denominado "Dejando atrás los modelos centrados en el déficit: desarrollo de un modelo estructural para explicar el clima social escolar. El rol del individuo, la familia, la comunidad y los valores desde un enfoque de desarrollo positivo adolescente", financiado por CONICYT y desarrollado entre noviembre de 2015 y noviembre de 2018.

${ }^{1}$ Doctor en Ciencias de la Educación. Universidad de La Frontera, Académico Departamento de Trabajo Social. Avenida Francisco Salazar 01145, Temuco, Chile. Tel.: +56-045-2325678. Correo: jose.galvez@ufrontera.cl

${ }^{2}$ Doctora en estadística. Universidad de La Frontera, Académica Departamento de Matemática, Chile.

${ }^{3}$ Doctor en Psicología. Departamento de Psicología. Universidad de La Frontera, Chile.

${ }^{4}$ Doctor en Psicología, Matemático. Universidad Pedagógica Nacional en Bogotá, Colombia.

${ }^{5}$ Magíster en Terapia Familiar Sistémica. Universidad de La Frontera, Chile. 


\section{Introducción}

Las actitudes hacia la autoridad formalmente establecida, resultan ser una variable de gran relevancia en el estudio del ajuste psicosocial de los adolescentes. Dicha relevancia, se ve contrastada con la baja cantidad de estudios psicométricos que analicen el efecto de la cultura sobre la medida de actitudes hacia la autoridad institucional (Cava, Estévez, Buelga, \& Musitu, 2013; Gálvez-Nieto, Vera-Bachmann, Trizano, \& García, 2015).

\section{Actitud hacia la autoridad institucional:} definición y variables asociadas

El constructo actitud hacia la autoridad institucional, se define como la relevancia que los adolescentes confieren a los referentes de autoridad formalmente establecida y a las normas institucionales, existiendo autoridades de carácter informal como los padres o grupo de pares y las de tipo formal como leyes, policía y profesores (Cava et al., 2013; Estévez \& Emler, 2009; Molpeceres, Llinares, \& Bernad, 1999; Molpeceres, Lucas, \& Pons, 2000; Moreno, Estévez, Murgui, \& Musitu, 2009).

La adolescencia es una etapa donde el individuo resulta más vulnerable frente a la manifestación de conductas desadaptativas (Martorell, González, Ordóñez, \& Gómez, 2011). La percepción negativa de la familia, comunidad y de las principales normas sociales y autoridades, se asocia con transgresiones normativas (Molpeceres et al., 1999) y con el comportamiento delictivo, el cual está estrechamente relacionado con el rechazo a la escuela, la conducta antisocial y el consumo de drogas (Estévez \& Emler, 2010). También, la actitud desfavorable hacia la autoridad se relaciona significativamente con un mayor riesgo de consumo de alcohol y otras sustancias psicoactivas (Cava, Murgui, \& Musitu, 2008), así como una menor satisfacción vital (Molpeceres et al., 2000).

La actitud positiva hacia la transgresión institucional se asocia con problemas de integración en el aula, mayores conductas disruptivas, violentas y agresivas entre pares (Cava et al., 2013; Estévez \& Emler, 2011; Estévez, Murgui, Moreno, \& Musitu, 2007; Moreno et al., 2009). De igual forma, la percepción negativa tanto de la escuela como de los docentes se liga con la manifestación de comportamientos agresivos hacia éstos (Moreno, Ramos, Martínez, \& Musitu, 2010), lo que conlleva a que los adolescentes sean sancionados por su comportamiento disruptivo, lo que aumenta su actitud desfavorable hacia las figuras de autoridad, constituyendo una pauta interaccional que se retroalimenta a través del tiempo (Moreno et al., 2010).

En consiguiente, se aprecia que adolescentes que presentan dificultades conductuales, establecen relaciones conflictivas con sus docentes (Cava, Buelga, Musitu, \& Murgui 2010; Estévez, Jiménez, \& Moreno 2011), observándose que éstos poseen una valoración negativa de la autoridad institucional, que se transforma en una actitud desfavorable hacia quienes la ejercen como hacia la institución en sí misma, tanto a nivel escolar como policial (Estévez \& Emler, 2009; Estévez, Martínez, \& Musitu, 2006; Molpeceres, et al., 1999; Moreno et al., 2009; Musitu, Estévez, \& Emler, 2007).

\section{Asociación entre actitud hacia la autoridad institucional y clima social escolar}

Como se abordará a continuación, uno de los contextos de gran influencia sobre el desarrollo adolescente y el aprendizaje de normas, es la escuela. La alta relevancia de este contexto está dada por la gran cantidad de tiempo que pasan los adolescentes en los establecimientos educacionales, aspecto que impacta directamente sobre el proceso de socialización (Orpinas \& Horne, 2006).

El clima social escolar se entiende como la percepción que los miembros de la institución escolar tienen respecto al ambiente en el cual desarrollan sus actividades habituales (Fan, Williams, \& Corkin, 2011; Hombrados-Mendieta \& Castro-Travé, 2013; Koth, Bradshaw, \& Leaf, 2008; Steffgen, Recchia, \& Viechtbauer, 2013; Thapa, Cohen, Guffey, \& Higgins-D’Alessandro, 2013; Trianes, Blanca, de la Morena, Infante, \& Raya, 2006), y está compuesto por todos aquellos factores físicos, elementos estructurales de la institución educativa (Cohen, McCabe, Michelli, \& Pickeral, 2009; López, Bilbao, \& Rodríguez, 2011). Diversos estudios han planteado que una actitud transgresora hacia la autoridad del 
profesor, se asocia con comportamientos disruptivos respecto de las leyes, normas e instituciones que rigen los comportamientos adecuados en la sociedad (Cava et al., 2010; Cava et al., 2013; Estévez \& Emler, 2009; Molpeceres et al., 1999). En estudios recientes se ha demostrado que la actitud hacia la autoridad institucional, se relaciona de manera positiva y directa con el clima social escolar (Gálvez-Nieto et al., 2015; Moreno, et al., 2009), con relaciones sociales apropiadas entre estudiantes (GarcíaHierro \& Cubo, 2009; Giovazolias, Kourkoutasb, Mitsopouloua, \& Georgiadib, 2010; LaRusso \& Selman, 2011; Steffgen et al., 2013) y con la reducción de conductas de riesgo (LaRusso, Romer, \& Selman, 2008).

\section{La escala de actitud hacia la autoridad institucional en adolescentes (AAIA): análisis de su estructura teórica y empírica}

La medida de actitudes hacia la autoridad institucional $(A A I-A)$ es un instrumento que evalúa el grado de aceptación o rechazo hacia los referentes de autoridad formalmente establecida (Cava et al., 2013). La AAI-A es una medida útil para la evaluación psicoeducativa en áreas vinculadas con la convivencia escolar, salud y prevención de la violencia escolar. En términos de prácticos, proporciona una medida precisa, fácil uso y número reducido de ítems, que facilita su aplicación tanto en contextos profesionales como en investigación académica.

La estructura teórica de la escala $A A I-A$ posee dos dimensiones; actitud positiva hacia la transgresión y actitud positiva hacia la autoridad. Dicha estructura teórica, fue puesta a prueba en un estudio psicométrico realizado simultáneamente en España y México (Cava et al., 2013), los resultados de esta investigación ratificaron empíricamente que la estructura teórica propuesta -dos factores inversamente correlacionados- fue la que mejor ajustaba a los datos. Otro estudio psicométrico desarrollado recientemente en Chile, confirmó dicha estructura factorial y presentó evidencias de validez de criterio externo analizando el grado de asociación con el clima social escolar (Gálvez-Nieto et al., 2015).

Como se expuso anteriormente, la AAI-A cuenta con evidencias psicométricas en España, México y Chile, estos estudios han demostrado la estabilidad de la estructura teórica de la escala. Si bien la $A A I-A$ ha demostrado su utilidad en diversos contextos, entre ellos, uno de los países comparados en esta investigación, es pertinente señalar que la validez no es una propiedad estática, sino que una propiedad dinámica que debe ser resguardada a partir de diferentes estudios y muestras, que avalen la robustez de las puntuaciones en términos de estabilidad factorial y precisión, utilidad práctica y correlación con otros constructos (Alvarado \& Santisteban, 2012).

Pese a la importancia de las actitudes hacia la autoridad institucional y su potencial impacto en el ámbito de la educación, este instrumento no registra estudios psicométricos en Colombia, tampoco estudios que analicen modelos estructurales simultáneos entre ambos países. En este contexto, un estudio comparativo cobra relevancia, pues ambos países comparten características importantes como el idioma y, en el caso de Chile, un aumento significativo en el flujo migratorio de población colombiana (Ministerio de Desarrollo Social, 2013).

Considerando estos antecedentes, este estudio tuvo como objetivo analizar el grado de equivalencia factorial, validez y fiabilidad de la escala de actitudes hacia la autoridad institucional $A A I-A$ en una muestra de adolescentes chilenos y colombianos. A partir de estos resultados, se espera contar con un instrumento validado en ambos países, que permita generar información contextualizada sobre la actitud hacia los referentes de autoridad, para la generación de programas de prevención de la violencia escolar y la promoción de un desarrollo saludable en la etapa adolescente.

\section{Método}

\section{Participantes}

Los participantes de la investigación fueron seleccionados a partir de un muestreo no probabilístico intencionado en 11 colegios públicos y particulares subvencionados de Chile y Colombia, totalizando 1,233 adolescentes; esta muestra no es representativa de la población adolescente, pero sí da cuenta de la variabilidad necesaria de los datos para estudios psicométricos, 
los cuales sugieren entre 5 y 10 participantes por ítem (Martínez-Arias, 1995).

Muestra chilena. Compuesta por 692 estudiantes adolescentes chilenos de ambos sexos (49.9\% hombres y $50.1 \%$ mujeres) con una edad promedio de 14.82 que osciló entre los 12 y 20 años $(D T=1.83)$. Estos estudiantes cursaban enseñanza secundaria en seis centros educativos de públicos y particulares subvencionados de la Región de la Araucanía, Chile.

Muestra colombiana: participaron 541 adolescentes colombianos de ambos sexos $(47.6 \%$ hombres y $52.4 \%$ mujeres) de edades comprendidas entre los 12 y $18 \quad(M=14.72$; $D T=1.26$ ). Los alumnos cursaban enseñanza secundaria en cinco establecimientos públicos en Bogotá, Colombia.

\section{Instrumentos}

Para desarrollar la investigación se utilizó la escala de actitudes hacia la autoridad institucional para adolescentes AAI-A y como medida convergente el cuestionario para evaluar clima social del centro escolar CECSCE.

La $A A I-A$ es una escala de autoinforme que mide las actitudes de los adolescentes hacia la autoridad institucional (Cava et al., 2013). Se compone de nueve ítems a los que se responde mediante una escala de cinco puntos $(1=$ Nunca, 5=Siempre). La AAI-A está compuesta por dos factores: Actitud Positiva hacia la Autoridad (APA), que se refiere al respeto hacia el profesorado y hacia la policía como figuras de autoridad. El segundo factor se denomina Actitud Positiva hacia la Transgresión (APT), entendido como la actitud positiva del estudiante hacia la transgresión de las reglas escolares y las normas legales. Las evidencias de confiabilidad y validez en España y México (Cava et al., 2013) y Chile (Gálvez et al., 2015) mostraron indicadores de ajuste psicométricos adecuados en términos de estructura factorial y fiabilidad. El último estudio realizado en Chile (Gálvez et al., 2015) mostró que el coeficiente alfa de Cronbach arrojó un valor de .712 para actitud positiva hacia la autoridad institucional (APA) y .751 para actitud positiva hacia la transgresión (APT).

El CECSCE es una escala de autoinforme elaborada originalmente en España (Trianes et al., 2006) y evalúa el clima social escolar desde la perspectiva de los estudiantes. Está compuesta por 14 ítems a los que se responde en una escala de cinco puntos $(1=$ Muy en desacuerdo, 5=Muy de acuerdo). Los estudios psicométricos realizados en Chile (Gálvez, Tereucán, Muñoz, Briceño, \& Mayorga, 2014; Gálvez-Nieto, Salvo, Pérez-Luco, Hederich, \& Trizano, 2017; Gálvez-Nieto, VeraBachmann, \& Trizano., 2015) evidencian que el CECSCE posee un adecuado ajuste psicométrico y dos factores. El primer factor se denomina Clima Social del Centro Escolar (CSE), que se refiere a las relaciones entre pares, capacidad de ayuda y sentimiento de bienestar. El segundo factor denominado Clima Social Profesores (CSP), evalúa exigencia académica, justicia y trato con los estudiantes. Los valores de fiabilidad del último estudio realizado en Chile (Gálvez et al., 2016), indican que el factor clima social escolar CSE obtuvo un coeficiente alfa de Cronbach igual a .810 , y el factor Clima Social Profesores CSP un alfa de Cronbach de .740.

\section{Procedimiento}

Se tomó contacto con los rectores de los establecimientos educativos y se solicitó autorización para aplicar los cuestionarios. Posteriormente se realizaron reuniones informativas con padres y apoderados, para obtener el consentimiento informado y resguardar los principios éticos del proyecto. Los estudiantes que accedieron a participar del estudio lo hicieron de manera voluntaria, anónima y firmaron un consentimiento informado.

Como la escala AAI-A no presentaba evidencias de validación en el contexto colombiano, los ítems de la escala se sometieron a revisión de pertinencia lingüística (tres jueces expertos). La selección de los jueces expertos se basó en dos criterios generales, conocimiento de la variable en estudio y conocimiento de la realidad cultural colombiana. Los resultados empíricos de esta revisión indicaron que los ítems cuatro y ocho debían ser modificados levemente, cambiando la expresión inicial "Es normal" por "Está bien".

\section{Análisis de datos}

Para entregar evidencias de invarianza factorial se realizaron distintos procedimientos de análisis de datos con las muestras de ambos 
países. Para asegurar la equivalencia de ambas muestras se calculó diferencias de proporción entre hombres y mujeres $\left(\chi^{2}(g l=1)=.622, p=.430\right)$ $\mathrm{y}$ diferencia de medias entre las edades $(t(g l=1231)=-1,155, \quad p=.248) \quad$ según país, no encontrándose diferencias estadísticamente significativas.

Para comprobar la invarianza factorial de la escala de actitudes hacia la autoridad institucional AAI-A entre las muestras de adolescentes chilenos y colombianos, se realizaron una serie de análisis factoriales confirmatorios mediante el software MPLUS 7.11 (Muthén \& Muthén, 2012). Para la estimación de los índices de bondad de ajuste, se utilizó la media y varianza ajustada del método de máxima verosimilitud robusta MLM (Muthén \& Muthén, 2012). Este procedimiento permite un análisis adecuado para datos que no cumplen con el supuesto de normalidad multivariante, como es el caso de los resultados del test de Mardia estandarizado en la muestra chilena $=14.758$ y la muestra colombiana $=13.442$ (Mardia, 1970). Dicha estimación permite además la obtención de índices robustos, así como estimaciones apropiadas de los parámetros y su nivel de error (Finney \& Di Stefano, 2006; Flora \& Curran, 2004). El modelo de análisis factorial confirmatorio fue analizado a través de los siguientes índices de bondad de ajuste; chicuadrado de Satorra Bentler $\left(S B-\chi^{2}\right)$ (Satorra \& Bentler, 2001), índice de ajuste comparativo (CFI), Tucker-Lewis Index (TLI) y Error cuadrático medio de aproximación (RMSEA). Para los primeros los índices CFI y TLI, se consideran como un ajuste adecuado del modelo, valores superiores a .90 (Schumacher \& Lomax, 1996), mientras que para el RMSEA, se considera un ajuste razonable valores inferiores a .08 (Browne \& Cudeck, 1993).

El análisis de invarianza factorial se realizó tomando los siguientes modelos (Vandenberg \& Lance, 2000): $M O$ - Invarianza de configuración, igual número de factores e ítems en ambos grupos; $M 1$-Invarianza métrica, igualdad entre las saturaciones factoriales; M2-Invarianza escalar, igualdad entre las intersecciones (medias de ítems iguales), M3-Invarianza de medias latentes, igualdad de las medias de los factores latentes. La comparación de los modelos se realizó tomando como criterio la diferencia de Chi-cuadrado de
Satorra Bentler $\triangle S B-\chi^{2}$ (Asparouhov \& Muthén, 2012) y el criterio de cambio de $\triangle C F I$ (Cheung \& Rensvold, 2002).

Una vez confirmada la solución factorial se evaluó la fiabilidad de los factores utilizando el Coeficiente alfa de Cronbach y el índice de homogeneidad ítem total, además considerando las limitaciones de este coeficiente (Dunn, Baguley, \& Brunsden, 2014; Green \& Yang, 2009; Revelle \& Zinbarg, 2009; Sijtsma, 2009; Trizano-Hermosilla \& Alvarado, 2016), dentro de las que se destaca el supuesto de tau-equivalencia o igualdad de saturaciones factoriales, el análisis de fiabilidad se verá complementado con el coeficiente omega (McDonald, 1999) entregando estimaciones más adecuadas de la fiabilidad al estimarse desde el modelo factorial.

\section{Resultados}

\section{Invarianza factorial entre adolescentes chilenos y colombianos}

El contraste de equivalencia factorial, de la Escala de actitudes hacia la autoridad institucional $(A A I-A)$ en estudiantes chilenos y colombianos, comenzó examinando la estabilidad de la estructura factorial en ambos países por separado. Para ello se estimaron dos modelos factoriales con los nueve ítems de la escala. El primer modelo de dos factores correlacionados para estudiantes chilenos reveló un ajuste satisfactorio, donde $S B \chi^{2}$ $(g l=26)=119.425, \quad p<.001 ; \quad C F I=.929 ; \quad T L I=.902 ;$ RMSEA $=.072$ (IC90\%=.059-.085). El segundo modelo correlacionado para estudiantes colombianos evidenció igualmente un ajuste adecuado, donde $S B-\chi^{2}(g l=26)=49.577, p<.001$; CFI $=.966 ; \quad$ TLI $=.954 ; \quad$ RMSEA $=.041$ (IC90\% $=.023-.058)$.

Estos índices ratifican que el modelo de dos factores (Cava et al., 2013; Gálvez et al., 2015) ajusta de forma adecuada a los datos, por lo que se confirma la estructura teórica planteada. Todas las cargas fueron estadísticamente significativas ( $p<.001)$. Además, se confirmó la correlación negativa entre los factores APA y APT tanto para Chile -.402 $(p<.001)$ como para Colombia -.305 $(p<.001)$.

El contraste de equivalencia factorial comenzó con un análisis preliminar en donde se examinó la bondad de ajuste de la estructura de la 
Tabla 1. Medias, desviaciones típicas (DT) y Análisis Factorial Confirmatorio

\begin{tabular}{|c|c|c|c|c|c|c|c|c|}
\hline \multirow[b]{3}{*}{ Ítems } & \multicolumn{4}{|c|}{ Muestra Chilena } & \multicolumn{4}{|c|}{ Muestra Colombiana } \\
\hline & \multirow[t]{2}{*}{ Media } & \multirow[t]{2}{*}{$D T$} & \multicolumn{2}{|c|}{ Cargas factoriales } & \multirow[t]{2}{*}{ Media } & \multirow[t]{2}{*}{$D T$} & \multicolumn{2}{|c|}{ Cargas factoriales } \\
\hline & & & APA & APT & & & APA & APT \\
\hline $\begin{array}{l}\text { 1.- Los profesores son justos a } \\
\text { la hora de evaluar. }\end{array}$ & 3.84 & .96 & $.682 *$ & & 3.80 & .84 & $.598 *$ & \\
\hline $\begin{array}{l}\text { 2.- La policía está para hacer } \\
\text { una sociedad mejor para todos. }\end{array}$ & 3.39 & 1.19 & $.467 *$ & & 3.15 & 1.04 & $.426^{*}$ & \\
\hline $\begin{array}{l}\text { 3.-Es normal saltarse la ley si } \\
\text { no se causa daño a nadie. }\end{array}$ & 2.58 & 1.24 & & $.511 *$ & 2.08 & 1.14 & & $.545^{*}$ \\
\hline $\begin{array}{l}\text { 4.-Los profesores tratan igual a } \\
\text { todos los estudiantes. }\end{array}$ & 3.27 & 1.30 & $.689 *$ & & 3.10 & 1.10 & $.607 *$ & \\
\hline $\begin{array}{l}\text { 5.- Si viese a alguien robar se lo } \\
\text { diría a la policía. }\end{array}$ & 3.97 & 1.29 & $.452 *$ & & 4.11 & 1.03 & $.487 *$ & \\
\hline $\begin{array}{l}\text { 6.- Estoy de acuerdo con lo que } \\
\text { hacen y dicen la mayoría de los } \\
\text { profesores. }\end{array}$ & 3.38 & 1.04 & $.698^{*}$ & & 3.33 & .78 & $.545^{*}$ & \\
\hline $\begin{array}{l}\text { 7.- Es normal desobedecer a los } \\
\text { profesores si no hay castigos. }\end{array}$ & 2.21 & 1.20 & & $.635^{*}$ & 1.77 & .92 & & $.823 *$ \\
\hline $\begin{array}{l}\text { 8.- Da igual saltarse las reglas } \\
\text { escolares si después no hay } \\
\text { castigos. }\end{array}$ & 2.24 & 1.26 & & $.857 *$ & 1.77 & .92 & & $.813^{*}$ \\
\hline $\begin{array}{l}\text { 9.- Si una regla escolar no te } \\
\text { gusta, lo mejor es saltártela. }\end{array}$ & 2.14 & 1.26 & & $.668^{*}$ & 1.73 & .95 & & $.717 *$ \\
\hline
\end{tabular}

Escala $A A I-A$ en la muestra chilena y colombiana (M0). Como se observa en la Tabla 2, los índices de ajuste $S B-\chi^{2}$, CFI, TLI y RMSEA de los modelos M0 para Chile y Colombia resultaron adecuados, siendo todos los parámetros estimados estadísticamente significativos.

Estos resultados confirmaron que la estructura de la escala $A A I-A$ de dos factores, con los mismos ítems, es identica para ambos países. Este modelo se consideró como referencia para la siguiente anidación de restricciones.

El siguiente modelo busca comprobar la invarianza métrica $(M I)$ agregando restricciones a las saturaciones de los ítems. Dicho modelo plantea como hipótesis la equivalencia de las cargas factoriales entre ambos grupos $\left(H_{x}: \Lambda_{x}{ }^{(\mathrm{Ch})}\right.$ $=\Lambda_{x}{ }^{(\mathrm{C})}$ ), donde $(\mathrm{Ch})$ representa el grupo chileno y (C) el grupo colombiano. Los índices de bondad de ajuste resultaron adecuados $S B$ $\chi^{2}(g l=59)=187.601 \quad p<.001 ; C F I=.937 ; T L I=.923 ;$ RMSEA $=.059$ (IC90\%=.050-.069), sin embargo al comparar este modelo con M0 se encuentran diferencias estadísticamente significativas, por lo que existen diferencias en al menos un ítem, entre las cargas factoriales de la escala de actitudes hacia la autoridad institucional en estudiantes chilenos y colombianos $\left(\Delta S B-\chi^{2}=16.20 \quad \Delta g l=7\right.$ $p=.023 ; \Delta C F I=-.004)$.
Considerando estos resultados se evaluó un modelo de invarianza métrica parcial (M1b) liberando la carga del ítem siete, debido a que presenta la mayor discrepancia de carga factorial entre los países. El modelo muestra adecuados índices de ajuste $\left(\Delta S B-\chi^{2}=8.64 \Delta g l=6 \quad p=.194\right.$; $\triangle C F I=-.001$ ), siendo muy similares a los índices de ajuste obtenidos en el $M O$, lo que indica que no hay diferencias entre el modelo de línea base $(M O)$ y el modelo con la restricción de las cargas factoriales $(M 1 b)$. Por consiguiente, no existen diferencias entre las cargas factoriales de las dos muestras evaluadas.

Al obtener adecuados índices de bondad de ajuste para los modelos $M 0$ y $M l b$, el siguiente paso fue comprobar si el Modelo 2 (M2) que impone la invarianza de cargas factoriales e interceptos. Como se observa en la tabla 2, este modelo presentó índices de ajuste adecuados, $S B$ $\chi^{2}(g l=65)=213.591 p<.001 ; C F I=.927 ; T L I=.919$; RMSEA $=.061 \quad(I C 90 \%=.052-.070)$, sin embargo, al comparar M2 con M1 se observan diferencias estadísticamente significativas $\left(\Delta S B-\chi^{2}=34.561\right.$ $\Delta g l=7 p<.001 ; \Delta C F I=-.014)$. De tal forma que la invarianza escalar, media de los ítems entre estudiantes chilenos y colombianos, no fue confirmada. Al no confirmarse la invarianza escalar (M2), no se puede estimar el modelo de invarianza de medias latentes (M3). 
Tabla 2. Índices de bondad de ajuste y comparación de modelos Chile y Colombia

\begin{tabular}{|c|c|c|c|c|c|c|c|c|c|}
\hline & $\mathrm{SB}-\chi^{2}(\mathrm{gl})$ & $\chi^{2} / \mathrm{gl}$ & TLI & CFI & $\begin{array}{l}\text { RMSEA } \\
(\text { IC90\%) }\end{array}$ & $\Delta \mathrm{SB}-\chi^{2}$ & $\Delta \mathrm{gl}$ & $\begin{array}{c}p \text {-valor } \\
\left(\Delta \mathrm{SB}-\chi^{2}\right)\end{array}$ & $\Delta \mathrm{CFI}$ \\
\hline $\begin{array}{l}1 \text { M0 Invarianza de } \\
\text { configuración }\end{array}$ & $171.379(52)$ & 3.295 & .919 & .941 & $.061(.051-.071)$ & & & & \\
\hline $\begin{array}{l}2 \text { M1 Invarianza } \\
\text { métrica }\end{array}$ & 187.601(59) & 3.179 & .923 & .937 & $.059(.050-.069)$ & 16.20 & 7 & .023 & -.004 \\
\hline $\begin{array}{l}3 \mathrm{M} 1 \mathrm{~b} \text { invarianza } \\
\text { métrica parcial }\end{array}$ & $180.489(58)$ & 3.112 & .925 & .940 & $.059(.049-.068)$ & 8.64 & 6 & .194 & -.001 \\
\hline $\begin{array}{l}4 \text { M2 Invarianza } \\
\text { escalar }\end{array}$ & $213.591(65)$ & 3.286 & .919 & .927 & $.061(.052-.070)$ & 34.561 & 7 & $<.001$ & -.014 \\
\hline
\end{tabular}

Nota: $S B-\chi^{2}=$ Chi-Cuadrado Robusto de Satorra y Bentler; $g l=$ grados de libertad; $T L I=$ índice de ajuste de Tucker-Lewis; $C F I=$ Índice de ajuste comparativo; $R M S E A=$ Error cuadrático medio de aproximación; $\Delta \mathrm{SB}-\chi 2=$ Cambio en Chi-Cuadrado Satorra y Bentler; $\Delta g l=$ cambio en grados de libertad; $\Delta C F I=$ Cambio en CFI.

$* p<.001$

Tabla 3. Fiabilidad. Correlación ítem total (C.I.T) Alfa de Cronbach si se elimina la sentencia (A.C.S)

\begin{tabular}{lcccc}
\hline & \multicolumn{2}{c}{ Chile } & \multicolumn{2}{c}{ Colombia } \\
\cline { 2 - 5 } Ítems & C.I.T & A.C.E & C.I.T & A.C.E \\
\hline 1.- Los profesores son justos a la hora de evaluar. & .527 & .670 & .427 & .520 \\
2.- La policía está para hacer una sociedad mejor para todos. & .435 & .700 & .319 & .573 \\
4.-Los profesores tratan igual a todos los estudiantes. & .531 & .661 & .392 & .532 \\
5.- Si viese a alguien robar se lo diría a la policía. & .412 & .713 & .286 & .590 \\
6.- Estoy de acuerdo con lo que hacen y dicen la mayoría de los profesores. & .565 & .653 & .409 & .533 \\
& $\alpha A P A=.726$ & $\alpha A P A=.604$ \\
3.-Es normal saltarse la ley si no se causa daño a nadie. & .456 & .753 & .423 & .750 \\
7.- Es normal desobedecer a los profesores si no hay castigos. & .545 & .705 & .587 & .644 \\
8.- Da igual saltarse las reglas escolares si después no hay castigos. & .687 & .624 & .597 & .638 \\
9.- Si una regla escolar no te gusta, lo mejor es saltártela. & .538 & .709 & .532 & .673 \\
\end{tabular}

\section{Análisis de fiabilidad}

Luego se realizó un análisis de fiabilidad por consistencia interna para Chile y Colombia mediante el coeficiente alfa de Cronbach y el coeficiente de omega de McDonal (señalado entre paréntesis). Para Chile, los resultados de fiabilidad del factor Actitud Positiva hacia la Autoridad arrojaron los siguientes valores .726 (.740) y para el factor Actitud Positiva hacia la Transgresión .757 (.768). Los resultados de fiabilidad para Colombia, para el factor Actitud Positiva hacia la Autoridad arrojaron los siguientes valores .604 (.670) y para el factor Actitud Positiva hacia la Transgresión .735 (.820).

En términos generales, la escala $A A I-A$ es entendida como una escala homogénea (véase tabla 3), los valores arrojados por el procedimiento correlación ítem total permiten señalar el aporte de cada uno de los ítems a la medición de un rasgo común. A continuación se presentan los resultados del análisis de fiabilidad en ambos grupos.

\section{Análisis correlacional}

Para evaluar la validez de criterio externo, se analizó la muestra completa y se correlacionaron las puntuaciones de la $A A I-A$ con los factores del CECSCE a través del coeficiente de correlación Rho de Spearman.

Todas las correlaciones fueron significativas $(p<.001)$ y en el sentido esperado, los factores clima social del centro escolar y clima social profesores, se correlacionan de manera negativa con la actitud positiva hacia la transgresión ( $r=-$ $254 ;-.271)$ y positivamente con la actitud positiva hacia la autoridad institucional $(r=.504 ; .631)$.

\section{Discusión}

El propósito de este artículo fue analizar la equivalencia y estabilidad psicométrica de la escala $A A I-A$ en estudiantes provenientes de Chile y Colombia. Los resultados permiten concluir que la $A A I-A$ presenta evidencia de calidad 
psicométrica para su utilización en los contextos de Chile y Colombia, además el grado de equivalencia transcultural del instrumento se presenta plausible a nivel de invarianza métrica parcial.

Los resultados del análisis factorial confirmatorio realizado de modo separado en la muestra chilena y colombiana ratifican la validez de la estructura teórica de dos factores inversamente relacionados. Los factores denominados "actitud positiva hacia la autoridad", referido a la actitud hacia profesores y policías como referentes de autoridad y "actitud positiva hacia la transgresión" relativo a normas escolares y actitud hacia la ley desde una perspectiva transgresora, demostraron ser la estructura teórica que mejor ajustaba a los datos en ambos grupos en estudio.

Por otra parte se analizó la invarianza factorial de la $A A I-A$ en ambas muestras de estudiantes. El primer modelo contrastado evidenció que el número de factores y los ítems que correspondían a cada factor, eran los mismos en la muestra de estudiantes chilenos y colombianos. El segundo modelo puesto a prueba entregó como resultado que las cargas factoriales de los ítems eran invariantes en ambas muestras liberando la carga del ítem siete. Lo anterior se conoce como invarianza parcial (Byrne, Shavelson, \& Muthen, 1989) que permite mayor flexibilidad que los modelos de invarianza completa. Estos resultados son coincidentes con el estudio transcultural realizado en población española y mexicana (Cava et al., 2013).

Es importante recordar que el objetivo de analizar el grado de invarianza factorial, es evaluar el grado de igualdad o equivalencia de la estructura teórica de la escala entre la muestra chilena y colombiana, en este sentido, las implicancias prácticas ante la falta de invarianza escalar, se manifiestan en la dificultad de realizar comparaciones válidas entre los promedios de los ítems y obviamente, en un grado más estricto, entre las comparaciones de las medias de las variables latentes de los factores de la escala.

Las evidencias de fiabilidad de la escala demuestran que los ítems poseen una consistencia interna adecuada, que facilita su aplicación en ambos contextos culturales. El análisis de homogeneidad estimado a partir del coeficiente de correlación ítem total corregido demostró adecuados niveles en cuanto al aporte de cada sentencia a la medición del constructo, sin embargo llama la atención el comportamiento del ítem cinco en la muestra colombiana "Si viese a alguien robar se lo diría a la policía" (C.I.T.=.286), según la opinión de expertos colombianos puede deberse al contenido del ítem y su significado negativo en el contexto cultural. $\mathrm{Al}$ respecto es necesario señalar que el punto de cohorte de .30 no es fijo, por ejemplo, Schmeiser y Welch (2006) sugieren una correlación ítem total de 20 .

Respecto de las evidencias de validez de criterio externo, la $A A I-A$ se correlaciona de manera significativa y en el sentido esperado con la medida de clima social escolar CECSCE. La evidencia teórica y empírica respalda que las actitudes hacia la autoridad institucional se relacionan con el clima social escolar (Cava et al., 2013; Estévez et al., 2011; Gálvez et al., 2015; Moreno et al., 2009).

Los resultados de esta investigación presentan el grado de estabilidad transcultural de la escala de actitudes hacia la autoridad institucional en adolescentes chilenos y colombianos, y su utilización en ambos contextos culturales. Entre las limitaciones de este estudio se encuentra la muestra, la cual, a pesar de ser amplia, debería ser probabilística y representativa de otras poblaciones que aporten evidencias para la generación de normas de baremación, para facilitar la interpretación de las puntuaciones de la escala. Así también, sería relevante poder desarrollar mayor cantidad de estudios transculturales en Latinoamérica aportando evidencia de comparabilidad y estabilidad de los constructos teóricos. Futuras líneas de investigación en América Latina podrían estudiar el papel de las actitudes hacia la autoridad institucional y sus relaciones con violencia escolar, familia o rendimiento académico. Del mismo modo sería interesante analizar posibles asociaciones con comportamientos predelictivos o drogadicción.

\section{Referencias}

Asparouhov, T., \& Muthén, B. (2013). Computing the strictly positive Satorra-Bentler chi-square 
test in Mplus. Mplus Web Notes: 12. Recuperado de http://www.statmodel.com/exa mples/webnotes/SB5.pdf

Alvarado, M. J., \& Santisteban, C. (2012). La validez en la medición psicológica. Madrid: UNED Ediciones.

Browne, M., \& Cudeck, R. (1993). Alternative ways of assessing model fit. En: Bollen, K. \& Long, J. (Eds.) Testing structural equation models. Beverly Hills, CA: Sage.

Byrne, M., Shavelson, R., \& Muthén, B. (1989). Testing for equivalence of factor covariance and mean structures: The issue of partial measurement invariance. Psychological Bulletin, 105(3), 456-466. doi: 10.1037/00332909.105.3.456

Cava, M., Buelga, S., Musitu, G., \& Murgui, S. (2010). Violencia Escolar entre adolescentes y sus implicaciones en el ajuste psicosocial: Un estudio longitudinal. Revista de Psicodidáctica, 15(1), 21-34.

Cava, M., Estévez, E., Buelga, S., \& Musitu, G. (2013). Propiedades psicométricas de la Escala de Actitudes hacia la Autoridad Institucional en adolescentes (AAI-A). Anales de Psicología, 29(2), 540-548.

Cava, M., Murgui, S., \& Musitu, G. (2008). Diferencias en factores de protección del consumo de sustancias en la adolescencia temprana y media. Psicothema, 20(3), 389395.

Cheung, G. W., \& Rensvold, R. B. (2002). Evaluating goodness-of-fit indexes for testing measurement invariance. Structural Equation Modeling, 9(2), 233-255. doi: 10.1207/S1532 8007SEM0902 5

Cohen, J., McCabe, L., Michelli, N., \& Pickeral, T. (2009). School Climate: Research, Policy, Teacher Education and Practice. Teachers College Record, 111(1), 180-213.

Dunn, T. J., Baguley, T., \& Brunsden, V. (2014). From alpha to omega: A practical solution to the pervasive problem of internal consistency estimation. British Journal of Psychology, 105(3), 399-412. http://doi.org/10.1111/bjop. 12046

Estévez, E., \& Emler, N. (2010). A Structural modelling approach to predict adolescent offending behaviour from family, school and community factors. European Journal on
Criminal Policy and Research, 16(4), 207220. doi: 10.1007/s10610-010-9128-5

Estévez, E., \& Emler, N. (2011). Assessing the links among adolescent and youth offending, antisocial behaviour, victimization, drug use and gender. International Journal of Clinical and Health Psychology, 11(2), 269-289.

Estévez, E., \& Emler, N. (2009). Individual differences in attitude to school and social reputation among peers: Implications for behavioural adjustment in educational settings. En J. E. Larson (Ed.), Educational Psychology. Nova.

Estévez, E., Jiménez, T., \& Moreno, D. (2011). Cuando las víctimas de violencia escolar se convierten en agresores: "¿Quién va a defenderme?". European Journal of Education and Psychology, 3(2), 177-186.

Estévez, E., Martínez, B., \& Musitu, G. (2006). La autoestima en adolescentes agresores y víctimas en la escuela: La perspectiva multidimensional. Intervención Psicosocial, 15(2), 223-232.

Estévez, E., Murgui, S., Moreno, D., \& Musitu, G. (2007). Estilos de comunicación familiar, actitud hacia la autoridad institucional y conducta violenta del adolescente en la escuela. Psicothema. 19(1), 108-113.

Fan, W., Williams, C., \& Corkin, D. (2011). A Multilevel Analysis of student perceptions of school climate: The effect of social and academic risk factors. Psychology in the Schools, 48(6), 632-647. doi: 10.1002/pits.20579

Finney, S., \& DiStefano, C. (2006). Non normal and categorical data in structural equation modeling. En G. Hancock \& R. Mueller (Eds.), Structural equation modeling. A second course (pp. 269-314). Greenwich, Ct: Information Age.

Flora, D., \& Curran, P. (2004). An empirical evaluation of alternative methods of estimation for confirmatory factor analysis with ordinal data. Psychological Methods, 9(4), 466-491. doi: 10.1037/1082989X.9.4.466

Gálvez, J. L., Tereucán, J., Muñoz, S., Briceño, C., \& Mayorga, C. (2014). Propiedades psicométricas del cuestionario para evaluar 
clima social del centro escolar (CECSCE). Liberabit, 20(1), 165-174.

Gálvez-Nieto, J. L., Salvo, S., Pérez-Luco, R., Hederich, C., \& Trizano-Hermosilla, I. (2017). Invarianza Factorial del Cuestionario para Evaluar Clima Social Del Centro Escolar (CECSCE) en estudiantes chilenos y colombianos. Revista Latinoamericana de Psicología, 49(2), 119-127. doi: 10.1016/j.rlp.2016.09.003

Gálvez-Nieto, J. L., Vera-Bachmann, D., Trizano, I., \& García, J. A. (2015). Examen psicométrico de la Escala de Actitudes hacia la Autoridad Institucional (AAI-A), en estudiantes chilenos. Revista Iberoamericana de Diagnóstico y Evaluación - e Avaliação Psicológica, 39(1), 57-67.

Gálvez-Nieto, J. L, Vera-Bachmann, D., \& Trizano., I. (2015). Estudio confirmatorio del Cuestionario para evaluar Clima Social del Centro Escolar en Chile. Revista Mexicana de Psicología, 32(2), 160-168.

García-Hierro, M., \& Cubo, S. (2009). Convivencia escolar en Secundaria: Aplicación de un modelo de mejora del clima social. Revista Electrónica Interuniversitaria de Formación del Profesorado, 12(1), 51-62.

Giovazolias, T., Kourkoutasb, E., Mitsopouloua, E., \& Georgiadib, M. (2010). The relationship between perceived school climate and the prevalence of bullying behavior in Greek schools: Implications for preventive inclusive strategies. Procedia Social and Behavioral Sciences, 5, 2208-2215.

Green, S. B., \& Yang, Y. (2009). Commentary on coefficient alpha: A cautionary tale. Psychometrika, 74(1), 121-135.

Hombrados-Mendieta, I., \& Castro-Travé, M. (2013). Apoyo social, clima social y percepción de conflictos en un contexto educativo intercultural. Anales de Psicología, 29(1), 108-122.

Koth, C. W., Bradshaw, C. P., \& Leaf, P. J. (2008). A multilevel study of predictors of student perceptions of school climate: The effect of classroom-level factors. Journal of Educational Psychology, 100(1), 96-104. doi: 10.1037/0022-0663.100.1.96

LaRusso, M., \& Selman, R. (2011). Early adolescent health risk behaviors, conflict resolution strategies, and school climate. Journal of Applied Developmental Psychology, 32(6), 354-362. doi: 10.1016/j.appdev.2011.05.003

LaRusso, M., Romer, D., \& Selman, R. (2008). Teachers as builders of respectful school climates: Implications for adolescent drug use norms and depressive symptoms in high school. Journal of Youth \& Adolescence, 37(4), 386-398. doi: 10.1007/s10964-0079212-4

López, V., Bilbao, M., \& Rodríguez, J. (2011). La sala de clases sí importa: Incidencia del clima de aula sobre la percepción de intimidación y victimización entre escolares. Universitas Psychologica, 11(1), 91-101.

McDonald, R. (1999). Test Theory: A unified treatment. Mahwah, NJ: Lawrence Erlbaum Associates.

Mardia, K. V. (1970). Measures of multivariate skewnees and kurtosis with applications. Biometrika, 57, 519-530. doi:10.1093/biomet/ 57.3.519

Martínez-Arias, R. (1995). Psicometría: teoría de los test psicológicos y educativos. Madrid: Síntesis

Martorell, C., González, R., Ordoñez, A., \& Gómez, O. (2011). Estudio confirmatorio del cuestionario de conducta antisocial (CCA) y su relación con variables de personalidad y conducta antisocial. Revista Iberoamericana de Diagnóstico y Evaluación - e Avaliação Psicológica, 31(1), 97-114.

Ministerio de Desarrollo Social (2013). CASEN: Inmigrantes, síntesis de resultados. Recuperado de http://observatorio.ministerio desarrollosocial.gob.cl/documentos/CASEN_ 2013_Inmigrantes_01_marzo.pdf

Molpeceres, M., Llinares, L., \& Bernad, J. (1999). La percepción de las figuras de autoridad formales e informales y la inclinación a la conducta delictiva en la adolescencia: Un análisis preliminar de sus relaciones. Intervención Psicosocial, 8(3), 349-367.

Molpeceres, M., Lucas, A., \& Pons, D. (2000). Experiencia escolar y orientación hacia la autoridad institucional en la adolescencia. Revista de Psicología Social, 15(2), 87-105.

Moreno, D., Estévez, E., Murgui, S., \& Musitu, G. (2009). Relación entre el clima familiar y el 
clima escolar: El rol de la empatía, la actitud hacia la autoridad y conducta violenta en la adolescencia. International Journal Of Psychological Therapy, 9(1), 123-136.

Moreno, D., Ramos, M., Martínez, B., \& Musitu, G. (2010). Agresión manifiesta y ajuste psicosocial en la adolescencia. SUMMA Psicológica, 7(2), 45-54.

Musitu, G., Estévez, E., \& Emler, N. (2007). Adjustment problems in the family and school contexts, attitude towards authority and violent behaviour at school in adolescence. Adolescence, 42(168), 779-794.

Muthén, L. K., \& Muthén, B. O. (1998-2012). Mplus User's Guide. Seventh Edition. Los Angeles, CA: Muthén \& Muthén.

Orpinas, P., \& Horne, A. (2006). Bullying prevention: Creating a Positive School Climate and Developing Social Competence. Washington D.C.: American Psychological Association.

Revelle, W., \& Zinbarg, R. (2009). Coefficients alpha, beta, omega, and the glb: Comments on Sijtsma. Psychometrika, 74(1), 145-154.

Satorra, A., \& Bentler, P. (2001). A scaled difference chi-square test statistic for moment structure analysis. Psychometrika, 66(4), 507514. doi:10.1007/BF02296192

Schmeiser, C. B., \& Welch, C. J. (2006). Test development. En R.L. Brennan (Ed.) Educational Measurement (4a ed. pp. 307353). Washington, DC: American Council on Education.

Schumacher, R., \& Lomax, R. (1996). A beginner's guide to structural equation modeling. New Jersey: Lawrence Erlbaum Associates, Publishers.

Sijtsma, K. (2009). On the use, the misuse, and the very limited usefulness of Cronbach's alpha. Psychometrika, 74(1), 107-120.

Steffgen, G., Recchia, S., \& Viechtbauer, W. (2013). The link between school climate and violence in school: A meta-analytic review. Aggresion and Violent Behavior, 18(2), 300309. doi: 10.1016/j.avb2012.12.001

Thapa, A., Cohen, J., Guffey, S., \& HigginsD'Alessandro, A. (2013). A review of school climate research. Review of Educational Research, 83(3), 357-385. doi: $10.3102 / 0034654313483907$
Trianes, M., Blanca, M., de la Morena, L., Infante, L., \& Raya, S. (2006). Un cuestionario para evaluar el clima social del centro escolar. Psicothema, 18(2), 272-277.

Trizano-Hermosilla, I., \& Alvarado, J. M. (2016). Best alternatives to Cronbach's Alpha reliability in realistic conditions: Congeneric and asymmetrical measurements. Frontiers in Psychology, 7, 1-8. doi: http://doi.org/10.3389 /fpsyg.2016.00769

Vandenberg, R., \& Lance, C. (2000). A review and synthesis of the measurement invariance literature: Suggestions, practices, and recommendations for organizational research. Organizational research methods, 3(1), 4-70. doi: 10.1177/109442810031002 